\title{
ANÁLISIS DE PATOLOGÍAS BUCALES HALLADAS EN ESTUDIOS REALIZADOS EN EL SERVICIO DE DIAGNÓSTICO POR IMÁGENES DE LA FACULTAD DE ODONTOLOGÍA DE LA UNIVERSIDAD NACIONAL DE CUYO
}

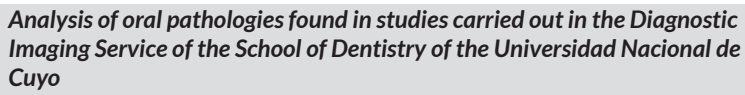

Analysis of oral pathologies found in studies carried out in the Diagnostic Imaging Service of the School of Dentistry of the Universidad Nacional de Cuyo

Fecha de Recepción: 20 de noviembre 2020
Análise de patologias bucais encontradas em estudos realizados no serviço de Diagnóstico por Imagem da Faculdade de Odontologia da Universidade Nacional de Cuyo

Aceptado para su publicación: 22 de febrero 2021

\section{Autores: \\ Paula Colomer ${ }^{1, a}$}

1. Anatomía y Fisiología Patológica. Diagnóstico por Imágenes I. Facultad de Odontología. Universidad Nacional de Cuyo. República Argentina. a. Especialista en Diagnóstico por Imágenes Buco-Máxilo-Facial. Universidad de Buenos Aires.

República Argentina.

Correspondencia: Colomer, Paula

Centro Universitario. M5502JMA. Mendoza, Argentina. + 542614135007

Correo electrónico: paula.colomer@outlook.com.ar

Conflicto de intereses: la autora declara no tener conflictos de interés.

Fuente de financiamiento: Secretaría de Investigación, Internacionales y Posgrado. Programa de becas de investigación y posgrado de la Universidad Nacional de Cuyo.

\section{Resumen}

La radiografía panorámica es uno de los exámenes radiográficos más utilizados en la clínica odontológica y es eficaz para evaluar la frecuencia y distribución de patologías bucales. Este estudio se realizó para evaluar la prevalencia de patologías bucales mediante el uso de radiografías panorámicas. Se llevó a cabo un estudio observacional correlacional en un corte transversal de tiempo, mediante el análisis de 490 estudios de radiografías panorámicas realizadas en el Servicio de Diagnóstico por Imágenes de la Facultad de Odontología de la Universidad Nacional de Cuyo (SID-Servicio de Imagen Diagnóstica) y se confeccionó un banco de datos sobre los hallazgos de las diferentes patologías maxilares y dentarias. La prevalencia de patologías dentales diagnosticadas por radiografías panorámicas fue del $65,6 \%$. Las anomalías de posición (49,1\%), fueron los subtipos más frecuentes de anomalías dentales. La radiografía panorámica puede ser una herramienta útil para la detección de patologías bucales, facilitando su derivación oportuna y/o una evaluación adicional.

Palabras clave: patología bucal; anomalías dentarias; diagnóstico por imagen; radiografía panorámica (fuente: DeCS BIREME).

\footnotetext{
Abstract

Panoramic radiography is one of the most commonly used radiographic examinations in the dental clinic and is effective in assessing the frequency and distribution of oral pathologies. This study was carried out to evaluate the prevalence of oral pathologies using panoramic $X$-rays. A correlational observational study was carried out in a cross-sectional time period by analyzing 490 studies of panoramic radiographs taken at the Diagnostic Imaging Service of the School of Dentistry of the Universidad Nacional de Cuyo (SID-Diagnostic Imaging
} 
Service) and a database was created on the findings of the different maxillary and dental pathologies. The prevalence of dental pathologies diagnosed by panoramic $X$-rays was $65.6 \%$. Position anomalies (49.1\%) were the most frequent subtypes of dental anomalies. Panoramic $X$-rays can be a useful tool for the detection of oral pathologies, facilitating timely referral and/or further assessment.

Key words: pathology, oral; tooth abnormalities; diagnostic imaging; radiography, panoramic (source: MeSH NLM).

\section{Resumo}

A radiografia panorâmica é um dos exames radiográficos mais amplamente utilizados na prática odontológica e é eficaz na avaliação da frequência e distribuição das patologias orais. Este estudo foi realizado para avaliar a prevalência de patologias orais por meio do uso de radiografias panorâmicas. Foi realizado um estudo observacional correlacional, em corte transversal no tempo, por meio da análise de 490 radiografias panorâmicas realizadas no Serviço de Diagnóstico por Imagem da Faculdade de Odontologia da Universidade Nacional de Cuyo (SID-Servicio de Imagen Diagnóstica) e um banco de dados foi preparado sobre os resultados das diferentes patologias maxilares e dentais. A prevalência de patologias dentais diagnosticadas por radiografias panorâmicas foi de 65,6\%. Anomalias posicionais $(49,1 \%)$ foram os subtipos mais frequentes de anomalias dentárias. A radiografia panorâmica pode ser uma ferramenta útil para a deteç̧ão de patologias orais, facilitando seu encaminhamento oportuno e / ou avaliação adicional.

Palavras-chave: patologia bucal; anormalidades dentárias; diagnóstico por imagen; radiografia panorâmica (fonte: DeCS BIREME).

\section{Introducción}

La radiografía panorámica (RP) es un estudio muy útil con múltiples posibilidades diagnósticas, permitiendo una exploración dental completa para así determinar el estado general de la cavidad bucal, puesto que nos ofrece la posibilidad de visualizar la totalidad de piezas dentales, así como también ver el estado en el que se encuentran el resto de ciertas estructuras, tales como los huesos maxilares o los componentes óseos de la articulación temporomandibular. Además, nos permite evaluar el desarrollo de los maxilares, revisar el estado en el que se encuentran los dientes temporarios y comprobar el proceso de erupción dental de los dientes permanentes, la presencia de anomalías dentarias, hallazgos de diferentes patologías maxilares y dentarias, lesiones quísticas, displásicas y tumorales ${ }^{1}$, entre otras alteraciones. De esta forma, queda claro que estamos ante uno de los estudios más recomendables a la hora de conseguir un diagnóstico precoz y general puesto que con tan solo una prueba, que se realiza en pocos minutos y de forma sencilla, podremos conocer la presencia de patologías no descubiertas hasta el momento².

Diferentes autores han realizado revisiones epidemiológicas de patologías maxilofaciales mediante $\mathrm{RP}^{3}$. Así, Goutham et al. ${ }^{4}$, analizaron la prevalencia de anomalías dentales, en la población de Odisha, India a través de RP y determinaron que las mismas estuvieron presentes en más de un tercio del grupo de estudio, llegando a la conclusión que el diagnóstico temprano ayuda a evitar complicaciones.

Por otra parte, Isola et al..$^{5}$, sostienen que se debe enfatizar en la necesidad de un análisis radiográfico de rutina, debido que a través del mismo se puede obtener un diagnóstico precoz de diversas patologías bucales. En este caso específicamente hacen referencia al odontoma que se presenta con mayor frecuencia en los huesos maxilares ${ }^{6}$, afirmando que es muy probable que la detección temprana del mismo sea un hallazgo radiológico accidental, y afirma también que un diagnóstico precoz en la dentición temporal es fundamental para prevenir complicaciones posteriores, como la impactación o el fracaso de la erupción de los dientes.

El objetivo de este estudio se realizó para evaluar la prevalencia de patologías bucales mediante el uso de RP.

\section{Materiales y Métodos}

Se realizó un estudio observacional retrospectivo, en un corte transversal de tiempo, mediante el análisis de 490 estudios de radiografías panorámicas realizadas en el Servicio de Diagnóstico de Ima- 
gen de la Facultad de Odontología de la Universidad Nacional de Cuyo (SID-Servicio de Imagen Diagnóstica) y se creó un banco de datos sobre los hallazgos de los diferentes patologías bucales.

Las RP, fueron tomadas con un ortopantomógrafo digital marca PLANMECA, con software Romexis $\AA$, en pacientes que habían tenido solicitud de estos estudios por derivación de colegas.

Se generó un archivo sistematizado de la prevalencia de anomalías y patologías de la región bucal, articulándose así con los objetivos del Observatorio de Salud Bucal de la Facultad de Odontología y con las líneas prioritarias de investigación de la institución.

Se llevó a cabo el análisis de dichos estudios, determinando la presencia de las alteraciones dentarias y óseas tales como neoplasias, lesiones quísticas y periapicales, anomalías dentarias, como también afecciones de la articulación temporomandibular. También se realizó un análisis estadístico de los hallazgos para establecer la prevalencia de los mismos.

\section{Resultados}

En la Figura 1, se observa el porcentaje de pacientes que presentaron algún tipo de patología, demostrando una diferencia significativa que justifica el estudio realizado ya que existían numerosas alteraciones sin registro alguno.

En la Figura 2, se observan las anomalías dentarias halladas, estratificadas en anomalías de número, tamaño, forma, estructura, erupción y posición, junto con su porcentaje de aparición en los estudios

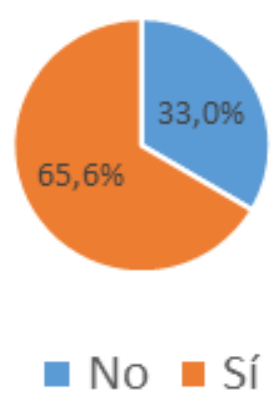

Figura 1. Patologías bucales. analizados, pudiendo observarse que las anomalías de posición fueron las más frecuentes. No se observaron diferencias de género.

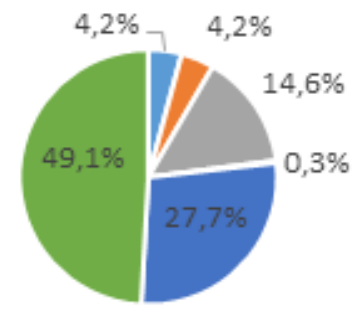

$$
\begin{aligned}
& \text { - Número | Tamaño | Forma } \\
& \text { - Estructura = Erupción = Posición }
\end{aligned}
$$

Figura 2. Anomalías Dentarias de Desarrollo.

En la Tabla I, además de registrar el porcentaje de las anomalías de desarrollo, también se puede observar el número de pacientes afectados.

Tabla I. Anomalías de desarrollo

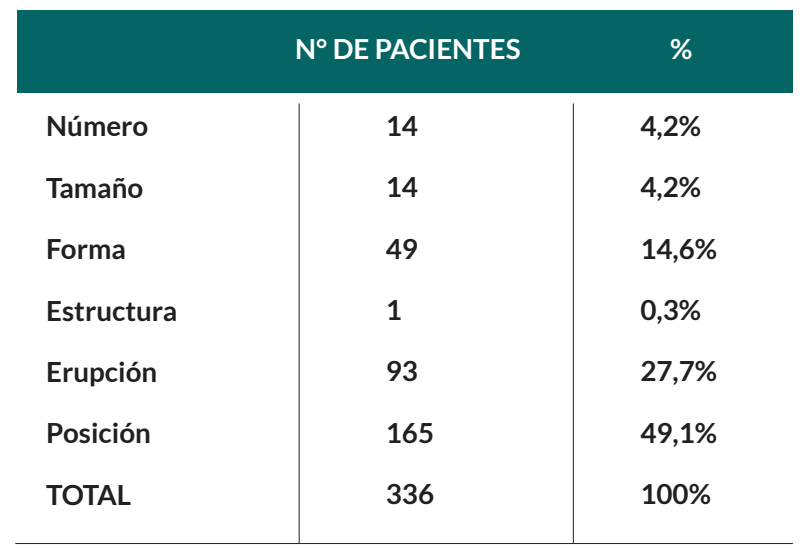

En la Figura 3, se muestra la cantidad de patologías bucales halladas en los diversos estudios de pacientes analizados, discriminando las diversas alteraciones y las más prevalentes.

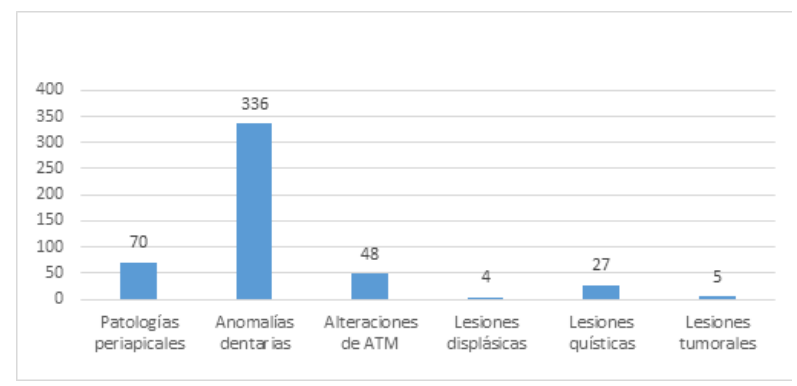

Figura 3. Patologías bucales. 


\section{Discusión}

La RP ha sido ampliamente analizada a través de diversos estudios epidemiológicos por ser un estudio radiográfico que se obtiene mediante un procedimiento rápido y sencillo y brinda múltiples beneficios, sobre todo la posibilidad de arribar a un diagnóstico precoz de diversas y múltiples patologías La prevalencia de lesiones bucales, anomalías dentales y patologías en diferentes poblaciones fue objeto de varios estudios ${ }^{7-11}$. En el presente trabajo, se encontraron lesiones dentales en su conjunto en $321(65,6 \%)$ pacientes de la muestra investigada.

Bekiroglu et al. ${ }^{12}$, analizaron 1056 RP en Turquía, concluyeron que desempeña un papel fundamental en el diagnóstico y la planificación del tratamiento de una amplia gama de enfermedades y afecciones dentales y maxilofaciales. En este estudio el $43 \%$ de los estudios radiográficos analizados presentaba algún tipo de patología, mientras que en nuestra investigación el porcentaje fue más elevado (65\%).

Ezoddini et al. ${ }^{13}$, las ortopantomografías de 480 pacientes, el 40,8\% presentaban anomalías dentarias, como dilaceración (15\%), dientes retenidos $(8,3 \%)$, taurodontismo $(7,5 \%)$ y dientes supernumerarios (3,5\%). Detectaron macrodoncia y fusión en algunas radiografías $(0,2 \%)$. Aunque en nuestro estudio no pudimos encontrar ninguna diferencia de género en cuanto a anomalías o patologías dentales, estos autores observaron que el $49,1 \%$ de los pacientes varones presentaban anomalías dentales frente al $33,8 \%$ de las mujeres.

También, la RP nos brinda información útil sobre la articulación temporomandibular, hallando en nuestro estudio numerosos casos de alteraciones de ATM que previamente no se habían registrado. Singh et al. ${ }^{14}$ sostiene que la combinación de la morfología anormal del cóndilo y muescas antegoniales acentuadas en una RP se correlaciona con la sinovitis de la ATM en la resonancia magnética, permitiendo predecir a través de la ortopantomografía alteraciones en la anatomía anormal que dan indicios de estar en presencia de una artritis idiopática juvenil ${ }^{15}$.

Aunque la RP debe utilizarse como complemento del examen clínico, tiene muchas ventajas en el examen oral, como la amplia cobertura de los dientes, la baja dosis para el paciente y el corto tiempo en el que se adquiere la imagen de la estructura facial, incluidos los huesos maxilares y mandibulares y sus estructuras de soporte 9,16 . Sin embargo, es importante tener en cuenta que la precisión diagnóstica de las patologías bucales en la RP es menor que la de las radiografías intraorales 17, pero esto requiere de más tiempo y recursos.

\section{Conclusiones}

A partir del presente proyecto de investigación se buscó concientizar a nuestros colegas sobre la importancia de la RP como estudio de rutina, previo a cualquier intervención. En muchos casos, se subestima el valor de la misma, se considera que "ha pasado de moda" ya que existen técnicas más innovadoras, pero es necesario tener presente que a través de dicho estudio se pueden prevenir complicaciones futuras al realizar diagnósticos precoces, con una simple toma radiográfica que hoy en día está al alcance de todos.

La ortopantomografía desempeña un papel fundamental en el diagnóstico y planificación del tratamiento de una amplia gama de enfermedades y afecciones dentales y maxilofaciales. Por lo tanto, esta técnica radiográfica, que si bien apareció hace más de 70 años, sigue teniendo vigencia en nuestros tiempos gracias a sus múltiples beneficios, siendo fundamental tenerla presente a la hora de iniciar cualquier tratamiento odontológico.

\section{Referencias Bibliográficas}

1. Soto L. Calero J. Anomalías en pacientes que asisten a la consulta particular e institucional en la ciudad de Cali 2009-2010. Colombia. Rev Estomat. 2010;1(1):17-23.

2. Saccucci M, lerardo G, Di Carlo G, Polimeni A, Sfasciotti GL. Marsupialization of radicular cyst in a 9-year-old child: report of a case and review of the literature. J Biol Regul Homeost Agents. 2013;27(2):603-6.

3. Kämmerer PW, Schneider D, Schiegnitz E, Schneider S, Walter C, Frerich B, Kunkel M. Clinical parameter of odontoma with special emphasis on treatment of impacted teeth-a retrospective multicentre study and literature review. Clin Oral Investig. 2016;20(7):1827-35. doi: 10.1007/s00784-015-1673-3

4. Goutham B, Bhuyan L, Chinnannavar SN, Kundu M, Jha K, Behura SS. Prevalence of Dental Anomalies in Odisha Population: A Panoramic Radiographic Study. J Contemp 
Dent Pract. 2017;18(7):549-553. doi: 10.5005/jp-journals-10024-2082

5. Isola G, Cicciù M, Fiorillo L, Matarese G. Association Between Odontoma and Impacted Teeth. J Craniofac Surg. 2017;28(3):755-758. doi: 10.1097/ SCS.0000000000003433

6. White S, Pharoah M. Radiología Oral. Principios e interpretación. Madrid: Ediciones Harcourt. 2002. 493-528. 7. Wagner VP, Arrué T, Hilgert E, Arús NA, da Silveira HLD, Martins MD, Rodrigues JA. Prevalence and distribution of dental anomalies in a paediatric population based on panoramic radiographs analysis. Eur J Paediatr Dent. 2020;21(4):292-298. doi: 10.23804/ejpd.2020.21.04.7

8. Pallikaraki G, Sifakakis I, Gizani S, Makou M, Mitsea A. Developmental dental anomalies assessed by panoramic radiographs in a Greek orthodontic population sample. Eur Arch Paediatr Dent. 2020;21(2):223-228. doi: 10.1007/s40368-019-00476-y

9. Laganà G, Venza N, Borzabadi-Farahani A, Fabi F, Danesi C, Cozza P. Dental anomalies: prevalence and associations between them in a large sample of non-orthodontic subjects, a cross-sectional study. BMC Oral Health. 2017;17(1):62. doi: 10.1186/s12903-017-0352-y

10. Garib DG, Lancia M, Kato RM, Oliveira TM, Neves LT. Risk of developing palatally displaced canines in patients with early detectable dental anomalies: a retrospective cohort study. J Appl Oral Sci. 2016;24(6):549-554. doi: 10.1590/1678-775720150535
11. Gabardo G, Maciel JVB, Franco A, de Lima AAS, da Costa TRF, Fernandes Â. Radiographic analysis of dental maturation in children with amelogenesis imperfecta: $A$ case-control study. Spec Care Dentist. 2020;40(3):267272. doi: $10.1111 /$ scd.12456

12. Bekiroglu N, Mete S, Ozbay G, Yalcinkaya S, Kargul B. Evaluation of panoramic radiographs taken from 1,056 Turkish children. Niger J Clin Pract. 2015;18(1):8-12. doi: 10.4103/1119-3077.146965

13. Ezoddini AF, Sheikhha MH, Ahmadi H. Prevalence of dental developmental anomalies: a radiographic study. Community Dent Health. 2007;24(3):140-4.

14. Singh S, Kumar S, Pandey R, Passi D, Mehrotra D, Mohammad S. Dimensional differences in mandibular antegonial notches in temporomandibular joint ankylosis. J Oral Biol Craniofac Res. 2011;1(1):7-11. doi: 10.1016/ S2212-4268(11)60004-3

15. Manfredini D, Lombardo L, Siciliani G. Temporomandibular disorders and dental occlusion. A systematic review of association studies: end of an era? J Oral Rehabil. 2017;44(11):908-923. doi: 10.1111/joor.12531

16. Bilge NH, Yeşiltepe S, Törenek Ağırman K, Çağlayan F, Bilge OM. Investigation of prevalence of dental anomalies by using digital panoramic radiographs. Folia Morphol (Warsz). 2018;77(2):323-328. doi: 10.5603/Fa2017.0087 17. Cantín M, Fonseca GM. Dens invaginatus in an impacted mesiodens: a morphological study. Rom J Morphol Embryol. 2013;54(3 Suppl):879-84. 